\title{
A compact and versatile dynamic flow cryostat for photon science
}

Peter J. E. M. van der Linden, Marco Moretti Sala, Christian Henriquet, Matteo Rossi, Kenya Ohgushi, François Fauth, Laura Simonelli, Carlo Marini, Edmundo Fraga, Claire Murray, Jonathan Potter, and Michael Krisch

Citation: Review of Scientific Instruments 87, 115103 (2016); doi: 10.1063/1.4966270

View online: https://doi.org/10.1063/1.4966270

View Table of Contents: http://aip.scitation.org/toc/rsi/87/11

Published by the American Institute of Physics

\section{Articles you may be interested in}

Towards 10 meV resolution: The design of an ultrahigh resolution soft X-ray RIXS spectrometer

Review of Scientific Instruments 87, 115109 (2016); 10.1063/1.4964847

Toroidal silicon polarization analyzer for resonant inelastic x-ray scattering

Review of Scientific Instruments 87, 083107 (2016); 10.1063/1.4959566

The simultaneous measurement of energy and linear polarization of the scattered radiation in resonant inelastic soft $x$-ray scattering

Review of Scientific Instruments 85, 115104 (2014); 10.1063/1.4900959

A precision cryostat design for manual and semi-automated cryo-plunge instruments Review of Scientific Instruments 87, 114302 (2016); 10.1063/1.4967864

A facility for the analysis of the electronic structures of solids and their surfaces by synchrotron radiation photoelectron spectroscopy

Review of Scientific Instruments 88, 013106 (2017); 10.1063/1.4973562

High energy-resolution $x$-ray spectroscopy at ultra-high dilution with spherically bent crystal analyzers of 0.5 $\mathrm{m}$ radius

Review of Scientific Instruments 88, 013108 (2017); 10.1063/1.4974100

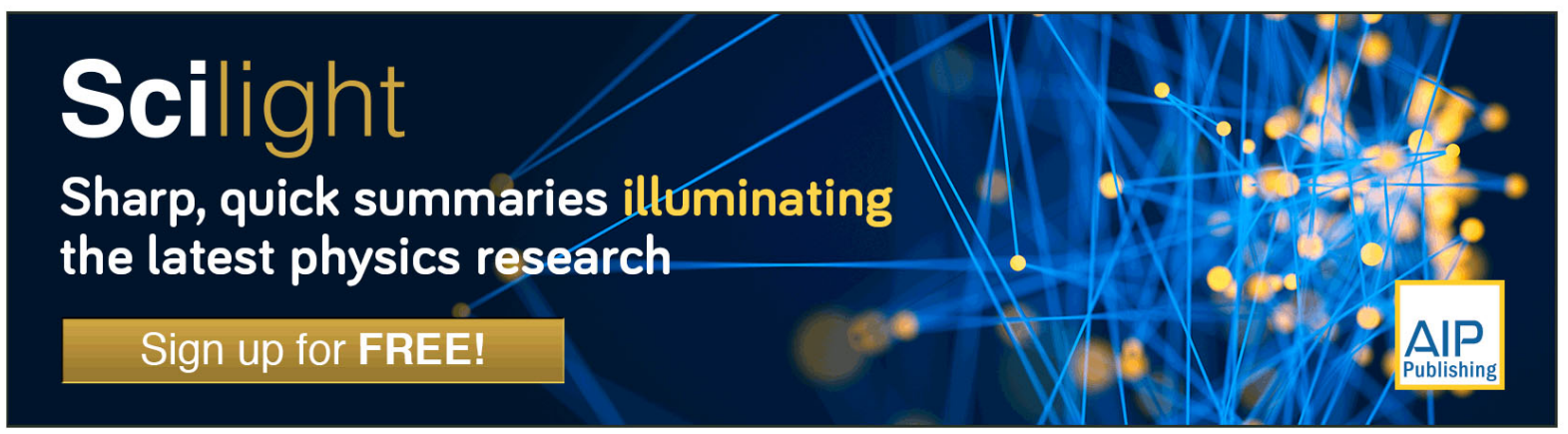




\title{
A compact and versatile dynamic flow cryostat for photon science
}

\author{
Peter J. E. M. van der Linden, ${ }^{1, a)}$ Marco Moretti Sala, ${ }^{1}$ Christian Henriquet, ${ }^{1}$ Matteo Rossi, ${ }^{1}$ \\ Kenya Ohgushi, ${ }^{2}$ François Fauth, ${ }^{3}$ Laura Simonelli, ${ }^{3}$ Carlo Marini, ${ }^{3}$ Edmundo Fraga, ${ }^{3}$ \\ Claire Murray, ${ }^{4}$ Jonathan Potter, ${ }^{4}$ and Michael Krisch ${ }^{1}$ \\ ${ }^{1}$ ESRF-The European Synchrotron, CS40220, 38043 Grenoble Cedex 9, France \\ ${ }^{2}$ Department of Physics, Graduate School of Science, Tohoku University, 6-3, Aramaki Aza-Aoba, Aoba-ku, \\ Sendai, Miyagi 980-8578, Japan \\ ${ }^{3}$ ALBA Synchrotron Light Source, Carretera BP 1413, Km. 3.3, 08290 Cerdanyola del Vallès, Barcelona, Spain \\ ${ }^{4}$ Diamond Light Source Ltd., Harwell Science and Innovation Campus, Didcot, \\ Oxfordshire OX11 ODE, United Kingdom
}

(Received 7 June 2016; accepted 10 October 2016; published online 8 November 2016)

\begin{abstract}
We have developed a helium gas flow cryostat for use on synchrotron tender to hard X-ray beamlines. Very efficient sample cooling is achieved because the sample is placed directly in the cooling helium flow on a removable sample holder. The cryostat is compact and easy to operate; samples can be changed in less than $5 \mathrm{~min}$ at any temperature. The cryostat has a temperature range of 2.5-325 K with temperature stability better than $0.1 \mathrm{~K}$. The very wide optical angle and the ability to operate in any orientation mean that the cryostat can easily be adapted for different X-ray techniques. It is already in use on different beamlines at the European Synchrotron Radiation Facility (ESRF), ALBA Synchrotron Light Facility (ALBA), and Diamond Light Source (DLS) for inelastic X-ray scattering, powder diffraction, and X-ray absorption spectroscopy. Results obtained at these beamlines are presented here. Published by AIP Publishing. [http://dx.doi.org/10.1063/1.4966270]
\end{abstract}

\section{INTRODUCTION}

Low temperature remains one of the most important tools to probe matter. Superconductivity, structural, electronic, and magnetic transitions, strongly correlated electron systems, study of lattice strains, and distortions are just few examples of scientific cases where low temperature contributes to the understanding of the complex balance between the competing interactions acting in a very large class of systems. Moreover, reducing the atomic thermal vibrations (by decreasing the temperature) results in a remarkable improvement of the resolving power of different $\mathrm{X}$-ray techniques.

Over the last two decades, a wide range of X-ray techniques has been developed and applied for research at low temperatures. However, with the continuously increasing brilliance of third generation synchrotron sources over the years, the resulting photon intensity renders cooling of samples to cryogenic temperatures on X-ray beamlines more difficult and critical. In this sense, the European Synchrotron Radiation Facility (ESRF) offers a good example: originally planned to reach a brilliance of $10^{18}$ photons $/ \mathrm{s} / 0.1 \% \mathrm{bw} / \mathrm{mm}^{2} / \mathrm{mr}^{2}$, this figure has now increased to above $10^{20}$ and the planned upgrade of the storage ring will yield another factor of 30 or more increase. At present, depending on the photon energy and beamline optics, the photon flux at the sample position covers a very wide range of $10^{9}$ to $10^{14}$ photons/s. At a photon energy of $6 \mathrm{keV}$, the X-ray beam power is between $1 \mu \mathrm{W}$ and $100 \mathrm{~mW}$. The resulting temperature increase of the sample at the beam spot depends on a number of factors. The size of the focal spot is of course of primary importance because it determines the

\footnotetext{
a) Author to whom correspondence should be addressed. Electronic mail: vanderlinden@esrf.fr
}

power density. The thermal conductivity of the sample defines how the heat spreads. The X-ray absorption in the sample itself and the sample container can vary from $100 \%$ to close to zero as a function of $\mathrm{X}$-ray energy and measurement method. The highest values are reached for techniques like X-ray absorption, Resonant Inelastic X-ray Scattering (RIXS) and Resonant $\mathrm{X}$-ray Emission Spectroscopy (REXS) which are done at an absorption edge, off a Bragg peak where the absorption would be low. At the bottom end, a diffraction experiment at energy above $15 \mathrm{keV}$ may have negligible absorption.

The incoming beam heat load can only be counterbalanced by efficient thermal anchoring of the sample to the cold source. In the case of a cryostat with the sample placed in the cryogenic insulation vacuum, the first bottleneck is the thermal conductivity of the sample itself and the second is its thermal contact with its container, substrate, and the cold source. Additional and more efficient sample cooling can be obtained by the use of contact gas. This is especially important for powder diffraction experiments: although the power absorbed in the sample and container is very small, the fact that the sample container consists of a glass capillary which is thermally isolating and needs to be rotated imposes the use of contact gas, either static or dynamic. The same holds for X-ray absorption spectroscopy (XAS) experiments in transmission mode where samples are diluted in X-ray transparent matrices of mediocre thermal conductivity such as, e.g., cellulose, or boron nitride or polyvinylpyrrolidone.

A static flow cryostat has a helium filled sample space which is separated from but thermalized to the cooling helium flow path. In a dynamic flow cryostat, the helium filled sample space is part of the cooling helium flow path. The sample is usually attached to a sample holder which is inserted into the sample space. The use of static exchange gas offers control of 
the exchange gas pressure but requires a separate system to pump and flush the sample space to avoid air pollution of the sample space upon sample change. Furthermore, the thermal conductance of the sample holder and gas column in a static flow cryostat can represent a considerable heat load on the cold side so the length of the sample space is usually increased to lower this head load. Even so, the preferred orientation of a static flow cryostat is vertically with the cold part on the bottom to avoid natural convection. As a dynamic flow cryostat uses forced convection, it can be designed with a much shorter sample space; moreover, it can be used in any orientation and has easier and quicker sample change than a static flow cryostat because no manipulation of exchange gas is needed.

The particular dynamic flow (DynaFlow) cryostat presented here was designed for operation on tender to hard X-ray beamlines. The sample is directly cooled by the helium flow. The cryostat is compact and light, has a modular construction, and can provide large angle optical access above $2 \theta=180^{\circ}$ in a plane either perpendicular or parallel to the axis of the cryostat. It provides the possibility for quick sample change at any temperature. The cryostat can operate in any orientation and accommodates different sample holders for different experiments; for example, fast spinning sample holders have been built for powder diffraction experiments. It reaches a minimum temperature below $2.5 \mathrm{~K}$ with helium consumption slightly above 2 liters of liquid per hour; when operated at $10 \mathrm{~K}$, the autonomy with a standard 1001 Dewar is more than a week. The cryostat can operate at any temperature up to $325 \mathrm{~K}$ with temperature stability better than $0.1 \mathrm{~K}$.

Three copies of the cryostat have been built. The original cryostat was designed for operation at the Inelastic X-ray Scattering beamline (ID20) of the ESRF. The two other copies were built for ALBA Synchrotron Light Facility (ALBA) beamlines Material Science Powder Diffraction (MSPD-BL04) and Core Level Absorption and Emission Spectroscopy (CLÆSSBL22), and for the High Resolution Powder Diffraction beamline (I11) at the Diamond Light Source (DLS).

\section{CONSTRUCTION, OPERATION, AND PERFORMANCE}

\section{A. Construction}

\section{Cryostat}

An overview of the cryostat construction is shown in Fig. 1. A peculiarity of this cryostat is the hybrid construction in metal and plastic. The central tube which is also the sample space consists of a stack of elements which are bolted together with indium seals to separate the cryogenic insulation vacuum from the helium flow circuit. The plastic parts are made of Torlon $\AA 4203 \mathrm{~L}$ polyamide-imide which is a standard grade material. Some selected cryogenic properties of Torlon are shown in Table I, together with a comparison with standard cryogenic materials. ${ }^{1-5}$ The thermal expansion is very small for a plastic and comparable to metals with large thermal expansion like brass and copper, and the mechanical properties are well above average compared to other plastics. The thermal conductivity on the other hand is roughly the same as for other plastics. The central Torlon tubes were designed to be very short with a big wall thickness; thus, they are sufficiently stiff to carry the weight of the copper heat exchangers and of lower thermal conductivity than a classical stainless steel structure would be while the short length diminishes the total cryostat length and thermal contraction. Correctly designed glue joints between Torlon and aluminium have proven to be reliable at cryogenic temperatures. As it is radiation hard, the material is also used as $\mathrm{X}$-ray window by machining the Inner Vacuum Chamber (IVC) to the thinnest possible section. The X-ray transmission was calculated using the average material composition found by nuclear magnetic resonance: ${ }^{6} 0.7 \times\left(\mathrm{O}_{4} \mathrm{~N}_{2} \mathrm{C}_{19} \mathrm{H}_{12}\right)+0.3$ $\times\left(\mathrm{O}_{3} \mathrm{~N}_{2} \mathrm{C}_{12} \mathrm{H}_{7}\right)$. In Fig. 2, the transmission of $1 \mathrm{~mm}$ of Torlon is compared with other commonly used window materials. Calculations were done using the XOP package. ${ }^{7}$ The sulfur $\mathrm{K}$ edge ( $2472 \mathrm{eV}$, i.e., the lowest edge accessible by the CLÆSS beamline) and $\mathrm{Ni} \mathrm{K}$ edge ( $8333 \mathrm{eV}$, intermediate energy range) are also reported for sake of comparison.

Starting from room temperature (Fig. 1(a), top left), the first element of the stack is the base flange with siphon entry, this is a one piece welded and brazed construction. The electric wiring connector and vacuum pumping port are positioned on the side of the base flange. The siphon entry receives an Oxford Instruments siphon which was chosen for its availability, reliability, and also because the icing of the helium pumping port occurs far away from the sample goniometry and its motors. The second element of the stack, the sample chamber base tube (Fig. 1(b)), is a Torlon cylinder screwed to the base with an indium joint at room temperature instead of the more usual Oring to gain some space and improve reliability.

The wall thickness of the base tube conditions the bending of the sample chamber under the weight of the copper heat exchangers when the cryostat is placed horizontally, but also the thermal conduction between room temperature and the secondary heat exchanger. The wall thickness was chosen quite large to limit the bending and give acceptable thermal performance. During operation, the helium gas in this part of the sample chamber forms a static column resulting in an additional heat load on the secondary heat exchanger.

The third element of the stack is the secondary heat exchanger (Fig. 1(c)) which is a crucial element as its efficiency determines the base temperature and helium consumption. It is made of Oxygen Free High Conductance (OFHC) copper and it is equipped with a Pt100 thermometer and heater. It was designed to intercept the incoming heat from room temperature as much as possible: as the exchanger cuts the central sample tube in two separate pieces, it intercepts the incoming heat from ambient particularly well. The secondary heat exchanger is clamped between two Torlon cylinders with two indium joints by a single set of brass screws (inset in Fig. 3). The use of brass ensures tightness of the indium seals due to its thermal expansion being larger than the copper of the heat exchanger. All of the wiring for the thermometers and heaters are thermally anchored to the secondary heat exchanger. An aluminium radiation shield (Fig. 1(g)) attached to the secondary heat exchanger covers the primary heat exchanger but may be extended to also cover the IVC. The fourth part of the stack is the sample chamber middle tube 


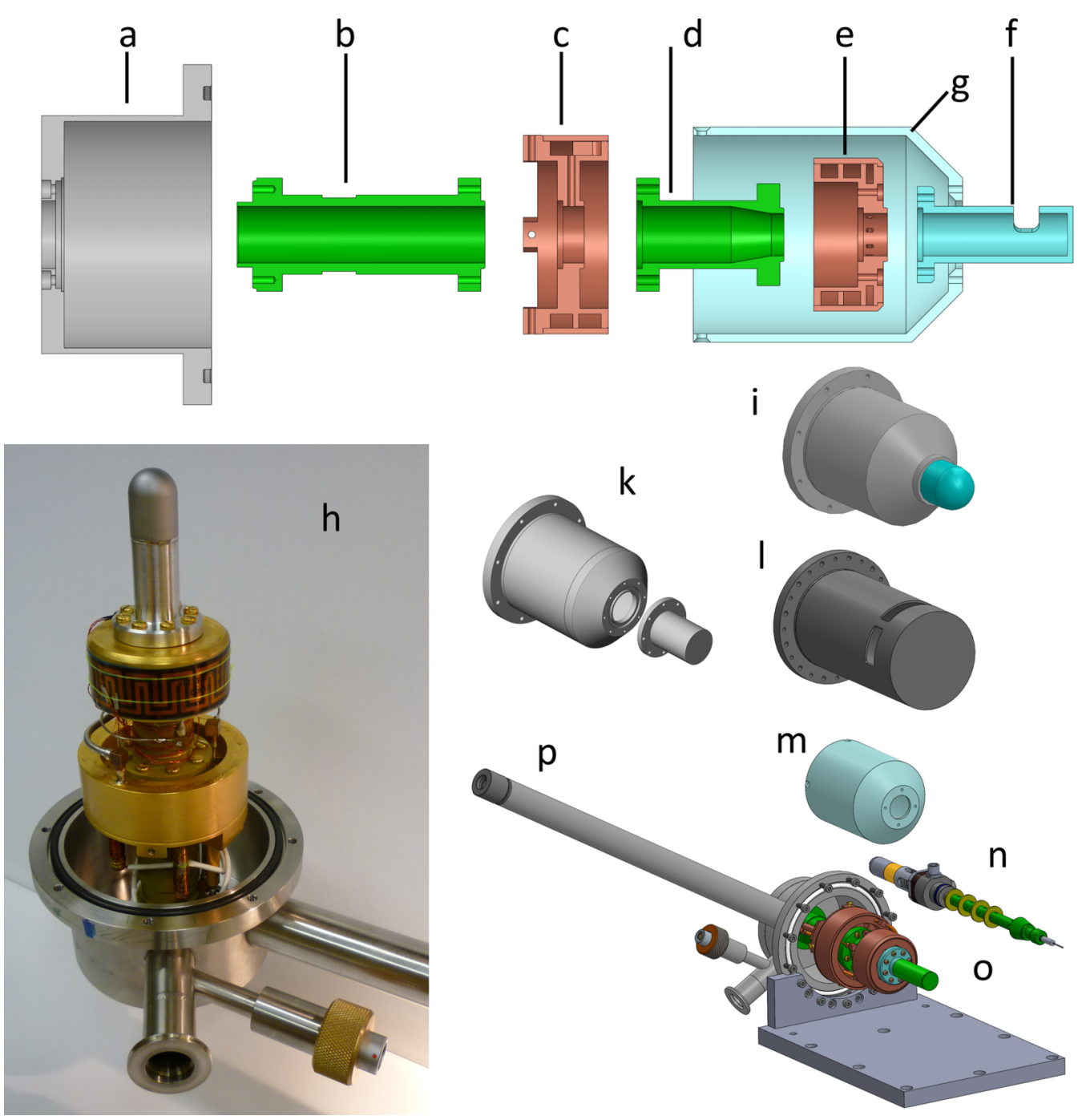

FIG. 1. Overview of the cryostat construction. Top panel: Cut view of the main cryostat components. (a): Base flange; (b): sample chamber base tube; (c): secondary heat exchanger; (d): sample chamber central tube; (e): primary heat exchanger; (f): inner vacuum chamber (IVC); (g): radiation shield. Bottom left panel (h): cryostat mounted and wired with beryllium dome IVC and without radiation shield. Bottom right panel: main cryostat components. (i): Outer vacuum chamber (OVC) with Be dome; $(\mathrm{k})$ : OVC in two pieces with $180^{\circ}$ window perpendicular to cryostat axis; (1): OVC in one piece; (m): OVC in two pieces with $180^{\circ}$ window parallel to cryostat axis; (n): radiation shield; (o): cryostat mounted on support and sample holder; and (p): siphon entry.

(Fig. 1(d)), a second Torlon cylinder situated in between the two heat exchangers. This part operates at much lower temperature; therefore, it has much lower thermal conductivity, and as it is additionally cooled by the helium gas flow it can be much shorter than the first cylinder. In practice, the length of the middle tube is made as short as possible.

The fifth part of the stack is the primary heat exchanger (Fig. 1(e)), also made of OFHC copper. It is equipped with a Cernox $₫$ thermometer and a Kapton $₫$ foil heater. The last part of the stack is the IVC, Fig. 1(f). Unlike the secondary heat exchanger, the two elements on both sides of the primary heat exchanger each have a set of screws (inset in Fig. 3). The IVC can thus be rapidly removed and replaced for repair or a change of window configuration. The sample space inside the IVC measures $14 \mathrm{~mm}$ diameter and $20-30 \mathrm{~mm}$ in length; the latter can easily be adapted to the requirements of the experiment. Various IVC's have been designed and tested in different material combinations: an all stainless chamber for cryogenic

TABLE I. Comparison of cryogenic material properties.

\begin{tabular}{lccccccccccc}
\hline \hline & \multicolumn{2}{c}{$\begin{array}{c}\text { Thermal expansion } \\
\left(\mathrm{K}^{-1}\right)\end{array}$} & & $\begin{array}{c}\text { Thermal } \\
\text { conductivity } \\
\left(\mathrm{W} \mathrm{m}^{-1} \mathrm{~K}^{-1}\right)\end{array}$ & $\begin{array}{c}\text { Yield } \\
\text { strength } \\
(\mathrm{MPa})\end{array}$ & $\begin{array}{c}\text { Tensile } \\
\text { modulus } \\
(\mathrm{GPa})\end{array}$ \\
\hline Temperature (K) & 4 & 77 & 300 & 4 & 77 & 300 & 77 & 300 & 77 & 300 \\
Torlon 4203L & $4.4 \times 10^{-7}$ & $1.3 \times 10^{-5}$ & $2.5 \times 10^{-5}$ & 0.013 & 0.1 & 0.25 & 218 & 192 & $\ldots$ & 4.9 \\
Stainless 304 & $-3.3 \times 10^{-7}$ & $6.6 \times 10^{-6}$ & $1.5 \times 10^{-5}$ & 0.29 & 7.8 & 15.3 & 1380 & 1034 & 200 & 193 \\
Copper & $2.8 \times 10^{-9}$ & $7.7 \times 10^{-6}$ & $1.7 \times 10^{-5}$ & 240 & 500 & 400 & 330 & 414 & 152 & 124 \\
Nylon & $-3.3 \times 10^{-7}$ & $3.2 \times 10^{-5}$ & $8.1 \times 10^{-5}$ & 0.013 & 0.29 & 0.34 & 207 & 55 & 6.9 & 2.1 \\
\hline \hline
\end{tabular}




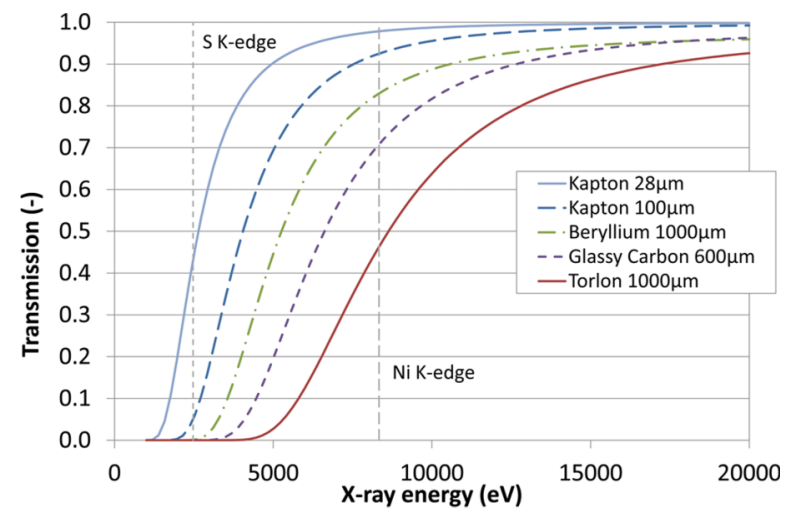

FIG. 2. Transmission as a function of energy for several commonly used window materials. Total material thickness is indicated in the inset, this corresponds to a window which is half as thick but is crossed twice by the X-rays.

testing, chambers combining an aluminium flange with beryllium or Torlon dome and an aluminium chamber with Kapton windows. An outer vacuum chamber (OVC, Fig. 1(k)) closes off the cryogenic insulation vacuum. The OVC has been built in one piece with cylindrical shape but also with a beryllium dome and as a two piece chamber to allow easier exchange of the part which has the windows.

\section{Helium flow circuit}

The helium flow circuit between the siphon arm and heat exchangers is made of $\mathrm{Cu} 70 / \mathrm{Ni30}$ tubing, diameter $0.6 \times 1 \mathrm{~mm}$ for the helium feed and $2.6 \times 3 \mathrm{~mm}$ for the pumping line. The tubing is soft soldered $(\mathrm{Pb} / \mathrm{Sn})$ to copper elbows to form the injection and pumping lines. Fig. 3 shows the cryostat flow scheme. Helium is supplied from a storage Dewar to the cryostat by the siphon inserted into the siphon arm. It passes through the primary heat exchanger where it is brought to the desired temperature and is then guided into the IVC. The sample holder is part of the helium flow path; helium enters into the IVC in front of the sample holder cone and is guided to pass by the sample; it then enters the sample holder central bore and flows backwards to exit the sample holder behind the cone. The cone thus separates the incoming and outgoing helium flow. Finally helium passes through the secondary heat exchanger and then leaves the cryostat.

\section{Sample holder}

The basic sample holder (Figs. 4(a) and 4(b)) consists of a room temperature flange with electrical connector for the thermometer and a series of Torlon cylinders glued together with radiation shields made of printed circuit board in between them. The choice of glue is not critical, as long as it is compatible with use at low temperature. Two types of glue have been used: Araldite 2020 and Stycast 2850 FT. There are no large forces on the sample holder, and all glue joints are far away from the $\mathrm{X}$-rays so radiation damage is not expected. The radiation shields also act as barriers against natural convection. The adapter of the sample holder in front of the cone is screwed in place, and different adapters have been developed for different experiments. When the sample holder is inserted into the cryostat, the cone of the sample holder sits on the cone of the sample chamber centre tube. An O-ring on the sample holder flange separates the helium flow circuit from ambient atmosphere. The flange also provides the possibility to attach a motor at the room temperature end (Fig. 4(c)). To remove the sample holder from the cryostat it is simply pulled out; during operation, it is held in place by the low pressure in the circuit. In order to maximize the efficiency in the limited available space, specific adapters were built to match different acquisition modes. Figs. 4(d) and 4(e) show two adapters for

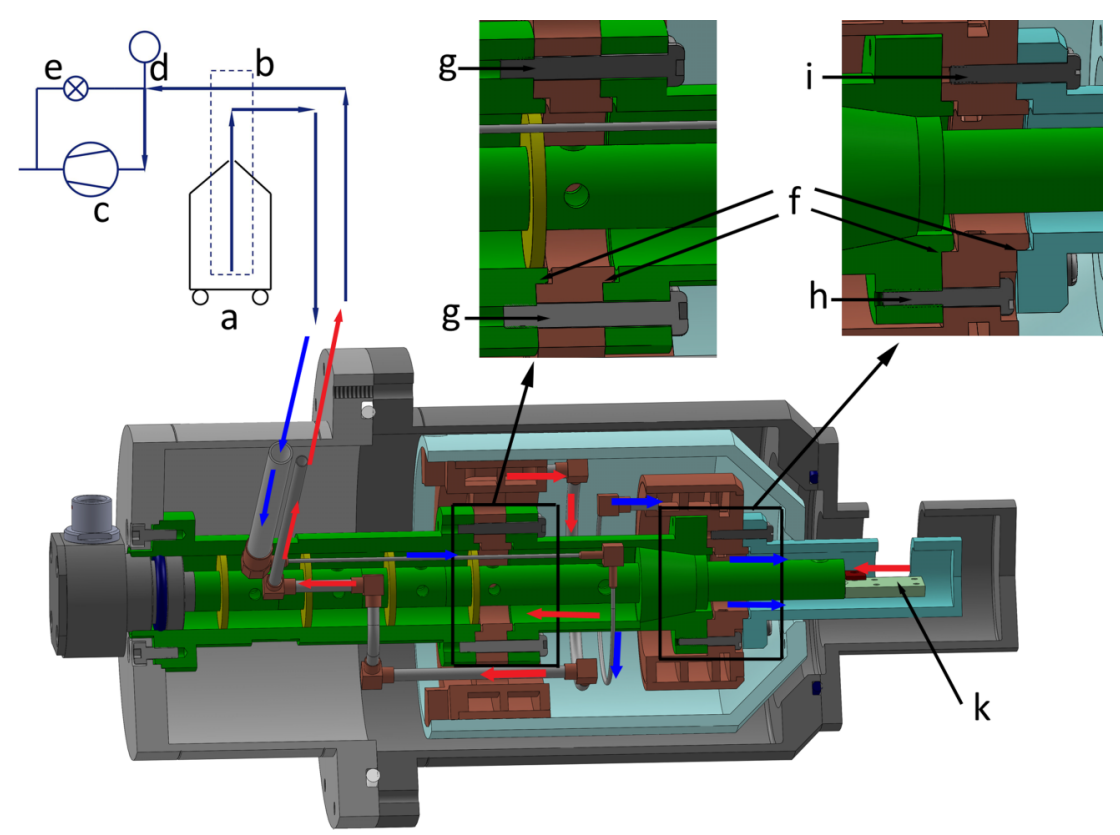

FIG. 3. Cryostat flow scheme. Arrows indicate the direction of helium flow. (a): Helium vessel. (b): siphon; (c): helium pump; (d): pressure gauge; (e): bypass valve; (f): positions of the indium joints; $(\mathrm{g})$ : a single set of screws clamps the secondary heat exchanger between two flanges; (h): screws to clamp the primary heat exchanger to the flange; (i): screws to clamp the IVC to the primary heat exchanger; and (k): sample position. 

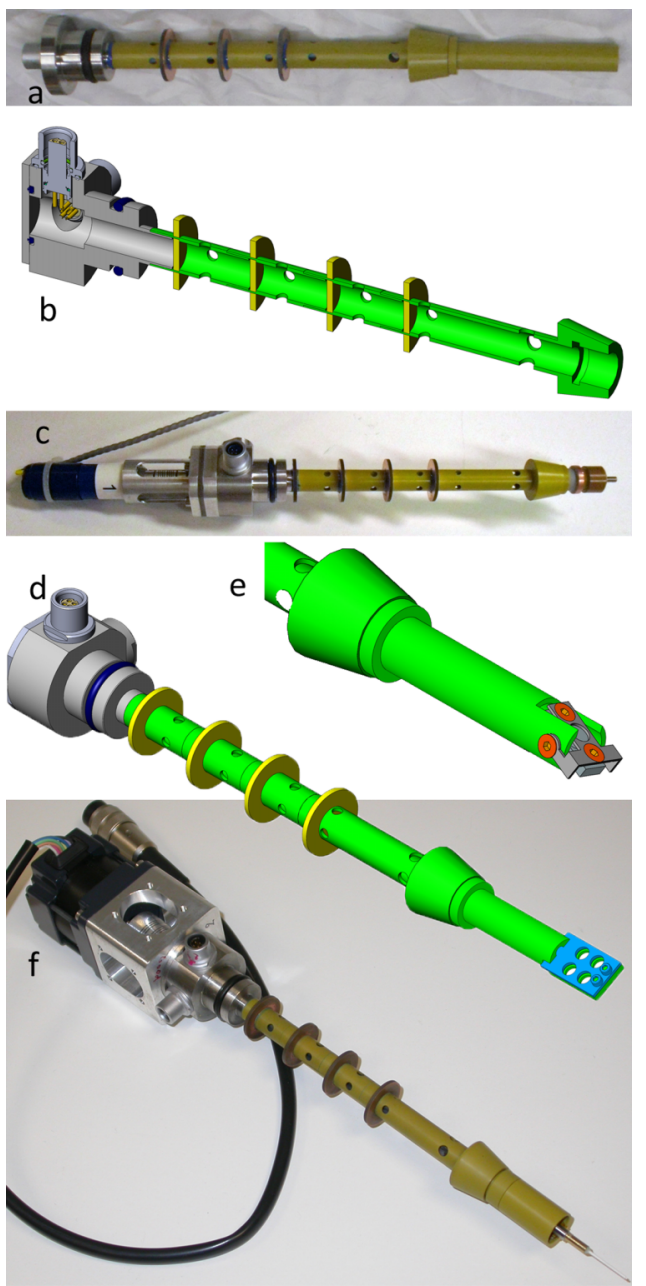

g

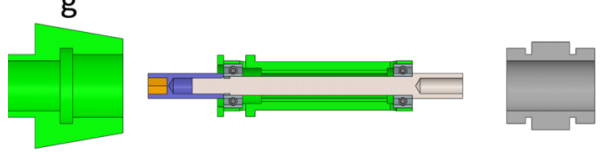

FIG. 4. Different sample holders. Top to bottom: (a): basic sample holder with a single on axis electrical connector; (b): cut view of basic sample holder with two electrical connectors for temperature and sample resistance; (c): sample spinner version DLS; (d): sample holders with adaptor four samples in XAS configuration; (e): adaptor for single sample XES configuration, angle is set and clamped with a screw; (f): sample spinner version ALBS; and $(\mathrm{g})$ : bearing cartridge of the ALBA spinner. Left to right the cone of the sample holder, the double bearing cartridge, and the clamp ring.

XAS and X-ray emission spectroscopy (XES) spectroscopy. For absorption measurements in transmission mode or emission spectroscopy, up to 4 samples (up to $5 \mathrm{~mm}$ each) can be placed simultaneously on the sample holder (Fig. 4(d)), reducing the number of sample changes and resulting in a more stable and reproducible temperature environment, which is especially important for in situ comparative studies. For absorption measurements in fluorescence mode, a single $5 \mathrm{~mm}$ sample adapter with adjustable angle is used (Fig. 4(e)). The angle between the sample surface and the incoming beam is set in polarisation dependent studies or to optimise the collection of the emitted light from the sample and to tune the illuminated sample portion.

For powder diffraction experiments, two different spinner sample holders were built (Figs. 4(c) and 4(f)). Both spinners include a rotation axis mounted at the room temperature side of the sample holder and penetrating into the IVC. At the room temperature side, the axis is driven by a motor while the vacuum tightness is guaranteed by a four lobe (JF4) O-ring. The DLS spinner version is equipped with a single hybrid ball bearing at the cold side and a single O-ring at the RT side, while the ALBA version is equipped with a double bearing cartridge at the cold side and an O-ring plus bearing at the RT side. The hybrid ball bearings consist of a $440 \mathrm{C}$ stainless steel cage and silicon nitride balls. The bearing cartridge (Fig. 4(g)) takes the place of the adaptor mentioned above. It consists of a static hollow shaft, a shaft clamp ring that screws into the sample holder, a two piece axle to clamp the bearings to the shaft, and a helium guide shield. The top part of the axle has a hexagonal imprint that clips into the hexagonal bottom of the driving axis. The cartridge provides a flow path for the helium gas and is easily removable for maintenance. The thermometer is fixed to the static shaft and can be removed without damaging its wiring. The ALBA spinner is capable of spinning up to $400 \mathrm{rpm}$ at a stable temperature of $5 \mathrm{~K}$.

\section{B. Operation and performance}

Before operation, the insulation vacuum has to be pumped to a suitable level. Experience shows that a vacuum level of $3 \times 10^{-5}$ mbar is sufficient; during cooling of the cryostat, the cryopumping effect will further reduce the pressure.

The choice of temperature controller and helium pump is not very critical. The tests were done with Lake Shore 336 and Cryo-con model $24 \mathrm{C}$ temperature controllers, and a Pfeiffer HENA25 rotary pump. The pumping line has to be sufficiently long so that helium enters the pump at ambient temperature to avoid damage to the pump. The base temperature depends on the size of the pump; a bigger pump will give a lower base temperature. Operation of the cryostat has also been tested without pump by pressurising the helium vessel. In this case, the base temperature was of course above $4.2 \mathrm{~K}$.

The cooldown of the cryostat usually takes 60-90 min. This is conditioned by the rather large thermal mass of the secondary heat exchanger. Fig. 5(a) shows a trace of a typical cooldown. The primary heat exchanger cools down to $25 \mathrm{~K}$ in $40 \mathrm{~min}$ while the secondary one remains above $200 \mathrm{~K}$. In this temperature range, the thermal conductivity of the Torlon sample chamber tube between the two heat exchangers is still quite high, and the resulting incoming power prevents the primary heat exchanger from going lower in temperature. As the secondary heat exchanger cools down, the thermal conduction of the sample chamber middle tube goes down and the primary heat exchanger also cools down further. At the very end of the cooldown, the temperature of the sample holder goes below the temperature of the heat exchanger. This was systematically observed for all three cryostats. Some liquid helium seems to arrive in the IVC and evaporates close to the sample, and with the shorter pumping path the temperature slightly drops. The helium consumption at base temperature is $2.2 \mathrm{l} / \mathrm{h}$. During one of the test cooldowns, the cryostat was oriented with the IVC downwards. Once the base temperature was reached, the cryostat was slowly rotated to the horizontal and vertical upward orientations without any observable change in temperature. 

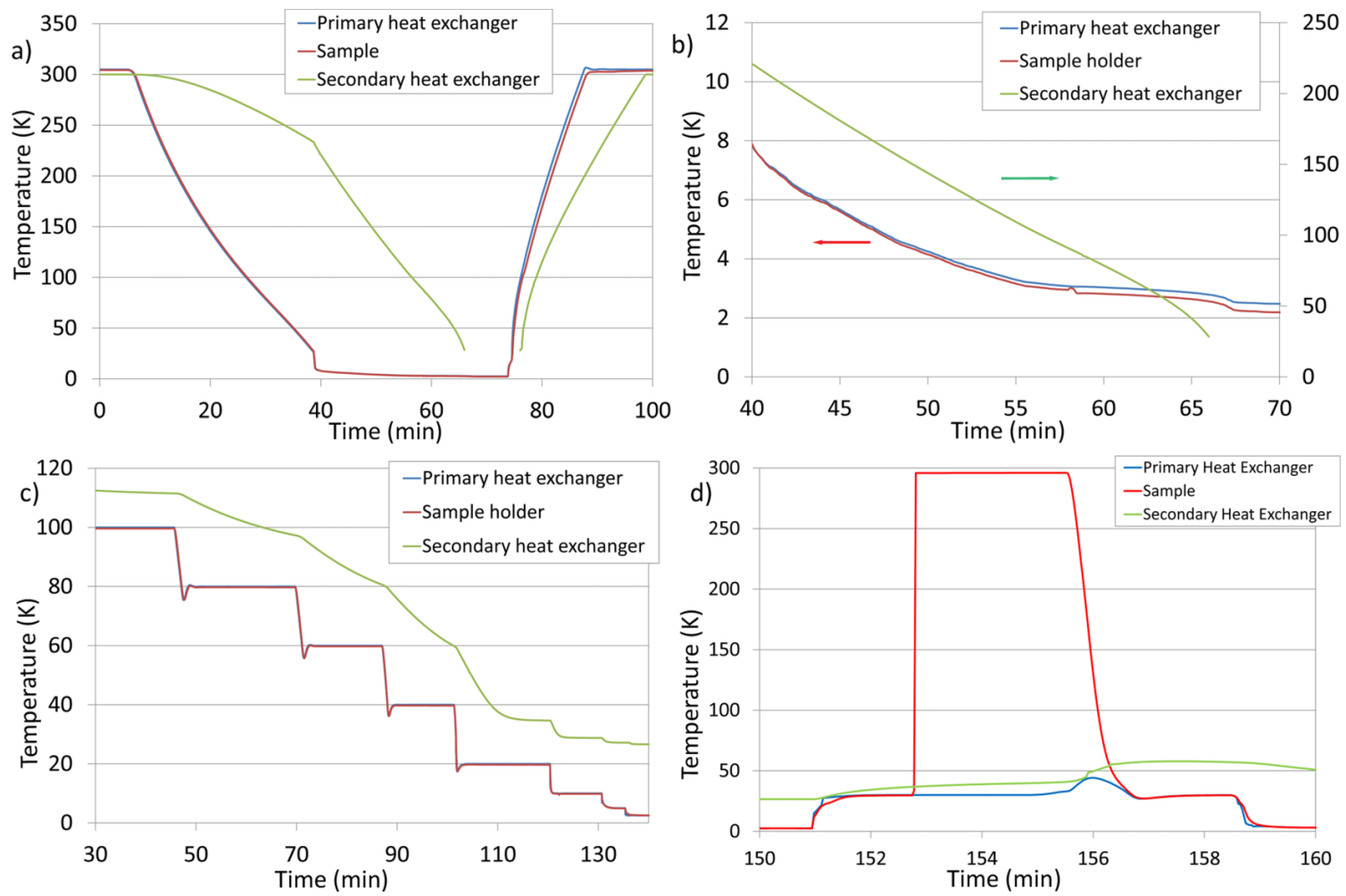

FIG. 5. Upper left panel (a): cooldown and warmup of the cryostat. The kink at $39 \mathrm{~min}$ is due to an increase of the helium flow. The calibration curve for the secondary heat exchanger temperature ends at $25 \mathrm{~K}$, below there is no valid reading. Upper right panel (b): last part of the cooldown of the cryostat. Bottom left panel (c): temperature regulation; the difference between the first heat exchanger and sample temperatures is too small $(<0.4 \mathrm{~K})$ to give a separation of the two curves. Bottom right panel (d): evolution of the temperature during sample change.

The temperature difference between the primary heat exchanger and sample holder visible in Fig. 5(a) still exists when the temperature of the cryostat is regulated at a constant value; this is due to the distance between these two parts. The temperature difference increases with temperature and decreases with helium flow rate. Choosing a lower flow rate will be more economical for the helium consumption but leads to long waiting times when stabilizing the temperature and it gives less efficient thermalisation of the sample.

Up to $40 \mathrm{~K}$, the thermal time constant of the cryostat is small and temperature regulation is fast. Above $40 \mathrm{~K}$, the primary heat exchanger and sample can still be brought to the desired temperature in a few minutes (see Fig. 5(b)) but the secondary heat exchanger has a long drift in temperature due to its large thermal mass. The resulting variation in the temperature gradient over the sample chamber base tube causes a drift in sample position due to thermal expansion. To counteract this effect, it is possible to use the second regulation loop of the temperature controller to set the temperature of the secondary heat exchanger to a value higher than it would naturally find in an equilibrium situation. One can appreciate inspecting Fig. 5(c) that this can be accomplished if the setpoint of the secondary heat exchanger was chosen $20 \mathrm{~K}$ above the setpoint of the primary.

To change the sample holder, the pump is stopped and the pressure in the circuit is brought up to atmospheric pressure by opening the bypass valve. The sample holder is pulled out and a replacement is plugged into the cryostat. During the time the cryostat is open, the overpressure in the helium return line will give a gas flow preventing air from entering. Insertion of the warm sample holder will bring a bit of air into the cryostat, but at the same time, the cold helium in the cryostat will warm up and expand in contact with the sample holder and drive the air into the pumping line. After placing the new sample holder, the bypass is closed and the pump restarted. This whole procedure can be done in a very short time limiting the risk of air entering the cryostat. Following changeover, the new sample will, of course, require some time to reach thermal equilibrium again. The whole sequence is shown in Fig. 5(d): at $t=151 \mathrm{~min}$, the setpoint is raised from base temperature to $25 \mathrm{~K}$. At $\mathrm{t}=153 \mathrm{~min}$, the connector of the sample temperature reading was plugged into the warm sample holder. From $t=153$ to $155 \mathrm{~min}$ some time is lost checking the sample. At $\mathrm{t}=155 \mathrm{~min}$, the cold sample holder is taken out and the warm sample holder is inserted. The temperature of the first heat exchanger goes up. The flow is restarted immediately and the sample holder temperature is stable at $25 \mathrm{~K}$ at $\mathrm{t}=158 \mathrm{~min}$. Sample holder change can be done at any operating temperature, but when the cryostat is running at very low temperature, the resulting thermal shock will often be much more damaging for the sample than for the cryostat and the risk of icing increases. In practice, the formation of ice has never been observed on any of the beamlines, probably also due to the limited duration of an experiment on an X-ray beamline (usually less than one week) and the small number of sample changes involved. 


\section{EXAMPLES OF SCIENTIFIC APPLICATIONS}

\section{A. ESRF ID20 use of the DynaFlow cryostat}

Beamline ID20 of the ESRF is dedicated to Inelastic Xray Scattering (IXS) techniques for the study of the electronic structure of materials. Temperature is one of the key control parameters in an IXS experiment, as shown in the following example of the use of the DynaFlow cryostat on ID20 reporting the case of resonant IXS (RIXS) on iridium oxides. At resonance, the incident photon energy is tuned to an absorption edge of the material under study. Many resonant processes can occur, but RIXS is often used to study low energy excitations, such as phonons, magnons, orbitons, or crystal-field and charge-transfer excitations, in which typical energy losses range from 0 to $10 \mathrm{eV}$. ID20 hosts a $2 \mathrm{~m}$ arm spectrometer, equipped with 5 spherical $\mathrm{Si}(844)$ diced crystal analysers, to analyse the energy of the scattered photons. A Si(111) double-crystal monochromator combined with a $\mathrm{Si}(844)$ back-scattering channel-cut monochromator is used to monochromatise the incident photons. The combined energyresolution of an $\mathrm{Ir}_{\mathrm{L}}$ edge RIXS experiment on ID20 is $25 \mathrm{meV}^{8}{ }^{8}$ We here focus on the study of magnetic excitations in $\mathrm{CaIrO}_{3}$, an antiferromagnetic insulator with post-perovskite structure. In these types of experiments, temperature control is crucial as it is necessary to lower the sample temperature well below the Néel temperature $\left(\sim 110 \mathrm{~K}\right.$ for $\left.\mathrm{CaIrO}_{3}\right)$ in order to observe magnetic excitations. Fig. 6 shows the temperature dependence of the RIXS spectra for a momentum transfer of $\boldsymbol{Q}=(1,2,9)$. Blue and red dots correspond to temperatures below $(20 \mathrm{~K})$ and above $(200 \mathrm{~K})$ the Néel temperature, respectively. The spectra show several interesting features at distinct energy losses: the elastic line at zero energy loss is much more pronounced in the spectrum taken at high temperature; the feature at $0.04 \mathrm{eV}$ and the broad distribution up to $0.30 \mathrm{eV}$ are associated to magnetic excitations. ${ }^{9}$ These develop into a resolution limited peak in the low temperature spectrum, when long-range antiferromagnetic order is established; the broad distribution of spectral weight between 0.40 and $0.80 \mathrm{eV}$, almost temperature independent, is instead associated to spinorbital transitions. ${ }^{9}$ It is clearly seen that temperature has a

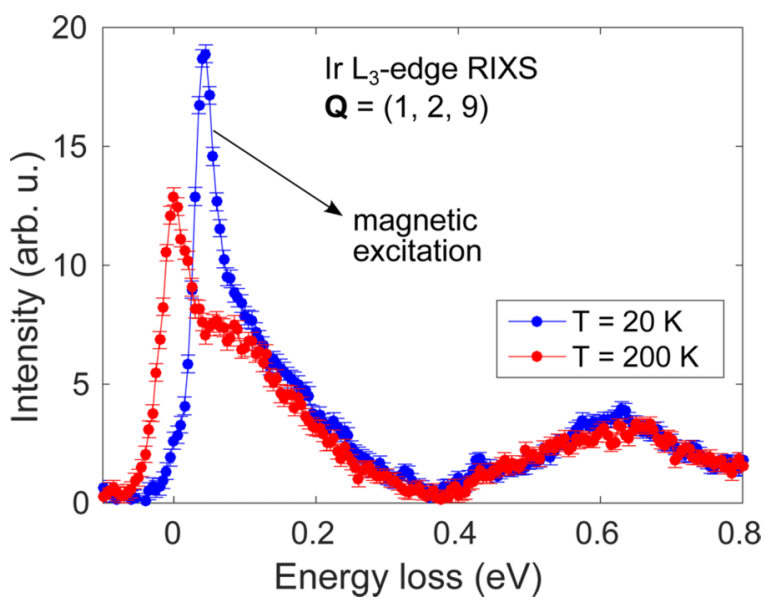

FIG. 6. RIXS spectra of $\mathrm{CaIrO}_{3}$ at a momentum transfer of $\boldsymbol{Q}=(1,2,9)$. Blue and red dots correspond to temperatures of $20 \mathrm{~K}$ and $200 \mathrm{~K}$, respectively. tremendous effect on the RIXS response, which can be directly linked to a change in the physical properties of the system under study.

\section{B. ALBA use of the DynaFlow cryostat}

At ALBA, use of the cryostat is shared between two beamlines, the Material Science Powder Diffraction beamline (BL04-MSPD) dedicated to powder diffraction crystallography and the Core Level Absorption and Emission Spectroscopy beamline (BL22-CLÆSS) dedicated to XAS/XES techniques. A detailed description of the MSPD beamline optics and experimental stations can be found in literature. ${ }^{10,11}$ Owing to the particular geometry of the BL04-MSPD Powder Station, the cryostat had to be compact enough in order to fit within a Huber 512.1-type Eulerian cradle (Fig. 7(a)), able to operate in horizontal orientation, and allow quick sample change whilst keeping the cryostat at low temperatures $(\sim 100 \mathrm{~K})$.

The CLÆSS beamline covers an unusually large energy range from 2.4 to $63 \mathrm{keV}$. The synchrotron radiation emitted by a multipole wiggler is first vertically collimated and then monochromatised by a $\mathrm{Si}(111)$ or $\mathrm{Si}(311)$ double crystal monochromator. A toroidal mirror focuses the radiation down to $100 \times 200 \mu \mathrm{m}^{2}$ at the sample position. Because of its compact design, the DynaFlow cryostat was also easily integrated in the CLÆSS experimental hutch, not only for the transmission and fluorescence absorption setups but also with the CLEAR emission spectrometer (Fig. 9(a)). Specific sample holders were designed and built at ALBA: spinning capillary for MSPD (Fig. 3(d)) and multi samples for CLÆSS (Figs. 3(b) and 3(c)).

In the following, first results obtained on MSPD and CLÆSS are presented. The background signal of the empty cryostat measured on MSPD (Fig. 7(b)) shows a peculiar pattern characterized at $10 \mathrm{~K}$ by an amorphous-like wide bump centred at $4.55 \AA \mathrm{d}$-spacing likely coming from the Torlon polymer and series of sharp Bragg peaks. Moreover, empty cryostat patterns collected at various temperatures exhibited slight changes in the peak positions. The only materials in the beam are the entrance/exit Kapton windows of the OVC and the Torlon walls of the IVC. The Kapton windows are thinner and situated far away from the diffractometer centre so the diffraction signal should be eliminated by the multianalyzer detection setup. The cryostat parasitic signal can be attributed to $\mathrm{TiO}_{2}$ which appears up to $3 \%$ in the Torlon 4203L composition. The Bragg reflections are nicely reproduced in LeBail type simulation using the rutile form of $\mathrm{TiO}_{2}$, space group $\mathrm{P}_{2} / \mathrm{mnm}$ with cell parameters $4.5937 \AA$ and $2.9587 \AA$ as starting values for refinement at room temperature.

Here it is important to note that because of the small diameter of the IVC, the multi-analyser setup cannot totally suppress the diffraction signal from the walls of the chamber. It means as well that with the actual Torlon chamber, the cryostat is not suitable for an "open" (non-collimated) detection setup as it is the case with the position sensitive detector MYTHEN. Empty cryostat measurements were performed with the latter setup and resulted in many intense diffraction peaks, arising 

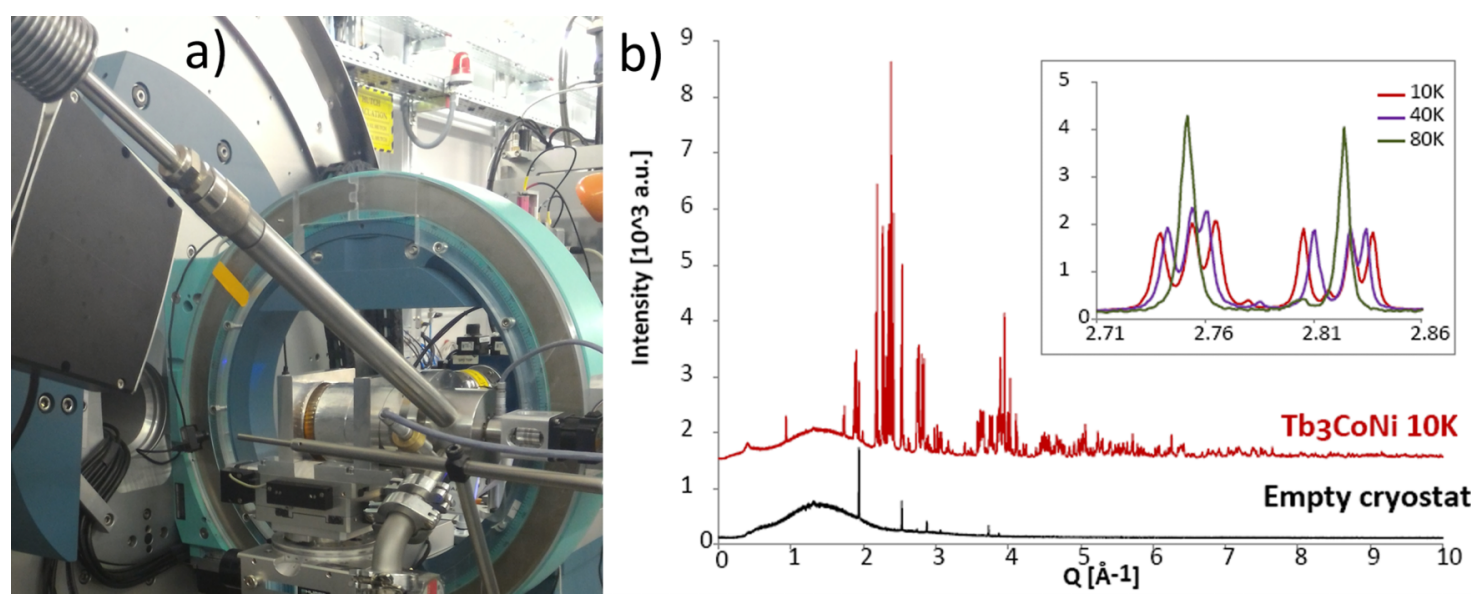

FIG. 7. (a) DynaFlow cryostat as mounted on the Eulerian cradle of the BL04-MSPD diffractometer. (b) Low temperature data of the empty cryostat and Tb3 $\mathrm{CoNi}$ at $10 \mathrm{~K}$. Data taken with the empty cryostat show a broad amorphous peak and a series of Bragg peaks at $4.80^{*}, 4.55,3.26,2.48,2.30^{*}, 2.18,2.05^{*}$, $1.68,1.62^{*}, 1.36^{*} \AA \mathrm{A}$-spacing (asterisk means intensity are ten times smaller on average). A selected angular range typical of the structural transition with data collected at 10,40 , and $80 \mathrm{~K}$ is magnified in the inset.

not only from the Torlon but also from the Kapton windows of the OVC. The option to have both the inner and outer chamber windows made of beryllium would dramatically reduce the $\mathrm{X}$ ray absorption, but these windows would have non negligible safety hazards and large financial consequences. They would also produce spurious Bragg peaks and the different trace elements would give large background signals at their respective edges would render fluorescence experiments very difficult.

Data were collected at $10 \mathrm{~K}$ at MSPD on the pseudo binary intermetallic compound $\mathrm{Tb}_{3} \mathrm{CoNi}$ which is part of the more general $\mathrm{R}_{3} \mathrm{Ni}_{2}$ series with $\mathrm{R}=\mathrm{Tb}$, Dy, Ho, Er, and $\mathrm{Y} .^{12,13} \mathrm{This}$ family of compounds is known to exhibit various crystal and magnetic structures depending on the rare earth ion, temperature, and/or Co doping. High resolution/high intensity neutron powder diffraction experiments were preliminarily performed on $\mathrm{Tb}_{3} \mathrm{CoNi}$ in order to determine the magnetic structure, and results tended to indicate a structural transition to occur at the onset of the magnetic transition $(\sim 100 \mathrm{~K})$. Due to a lack of angular resolution and strong magnetic scattering signal arising from the $\mathrm{Tb}^{3+}$ ions, it was however impossible to identify the reduction of symmetry from the high temperature hexagonal R-3 phase and unambiguously determine the magnetic structure. ${ }^{14}$ An initial synchrotron experiment was performed on MSPD using a commercial nitrogen blower device and data collected at the lowest possible temperature of $80 \mathrm{~K}$ further confirmed the possibility of a structural transition as evidenced by the broadening of selected peaks but it was still not possible to precisely determine the new space group. Measurements at lowest temperatures were therefore essential. Synchrotron powder diffraction measurements using the DynaFlow cryostat were performed at wavelength $\lambda=0.3180 \AA$ and with the sample confined in a $0.3 \mathrm{~mm}$ diameter borosilicate glass capillary. Both conditions were chosen to minimize absorption. The capillary was mounted on top of an ad-hoc sample holder designed and built at ALBA which allowed spinning the sample up to $1 \mathrm{~Hz}$ whilst collecting data, hence improving powder averaging. Despite the intrinsic cryostat signal problems mentioned before, diffraction patterns of the $\mathrm{Tb}_{3} \mathrm{CoNi}$ compound are fully exploitable down to $0.6 \AA \mathrm{d}$-spacing and a clear structural transition is observed below $80 \mathrm{~K}$ as can be seen in the inset in Fig. 7(b). The $10 \mathrm{~K}$ phase was refined using the Fullprof software package ${ }^{15}$ in a triclinic space group R-1 with unit cell angles $\alpha=90.054(1), \beta=90.698(1)$, and $\gamma=119.766(1)$. With the new space group information, it was then possible to successfully refine the neutron data and determine the magnetic structure of $\mathrm{Tb}_{3} \mathrm{CoNi}$. This consistsin accordance to magnetic symmetry analysis-mostly of ferromagnetic couplings on the five different rare earth sites which are not aligned to each other. Analysis of the general $\mathrm{Tb}_{3} \mathrm{Co}_{1-\mathrm{x}} \mathrm{Ni}_{\mathrm{x}}$ system is ongoing and will be published elsewhere but preliminary measurements presented here clearly demonstrate that high angular resolution synchrotron powder diffraction data collected at lowest possible temperatures are crucial for a complete understanding of the interplay between magnetic, structural, and electronic properties in transition metal and/or lanthanide based compounds.

When dealing with low temperature sample environments, it is always questioned what are the lowest significant temperatures achievable. In the case of the DynaFlow cryostat and powder diffraction, whereas it is clear that the gas in the sample chamber can reach $4 \mathrm{~K}$, it is not evident that samples in powder form and confined in low thermal conducting glass capillaries will effectively thermalize with the dynamic exchange gas. In the following, we demonstrate that structural anomalies could be detected down to $5 \mathrm{~K}$. The probed system was the $\mathrm{x}=0.25$ member of the multiferroic compound series $\mathrm{Mn}_{1-\mathrm{x}} \mathrm{Co}_{\mathrm{x}} \mathrm{WO}_{4}$ which is known to exhibit successive magnetic transitions below $40 \mathrm{~K}$. Further details of the scientific case can be found in Ref. 16 which reports results for the two parent compounds $\mathrm{x}=0.05$ and 0.20 . Based on both synchrotron powder diffraction experiments at the ESRF powder diffraction beamline and magnetic susceptibility measurements, subtle lattice anomalies $\left(\Delta \mathrm{l} / 1 \sim 10^{-5}\right)$ were noticed coinciding with the magnetic transitions. $\mathrm{Mn}_{75} \mathrm{Co}_{0.25} \mathrm{WO}_{4}$ data were collected on BL04-MSPD at wavelength $\lambda=0.4129 \AA$ down to $5 \mathrm{~K}$ with narrow temperature steps in the 5-30 K range. Fig. 8 displays selected unit cell parameters refined from collected patterns together with magnetic susceptibility measurements which 


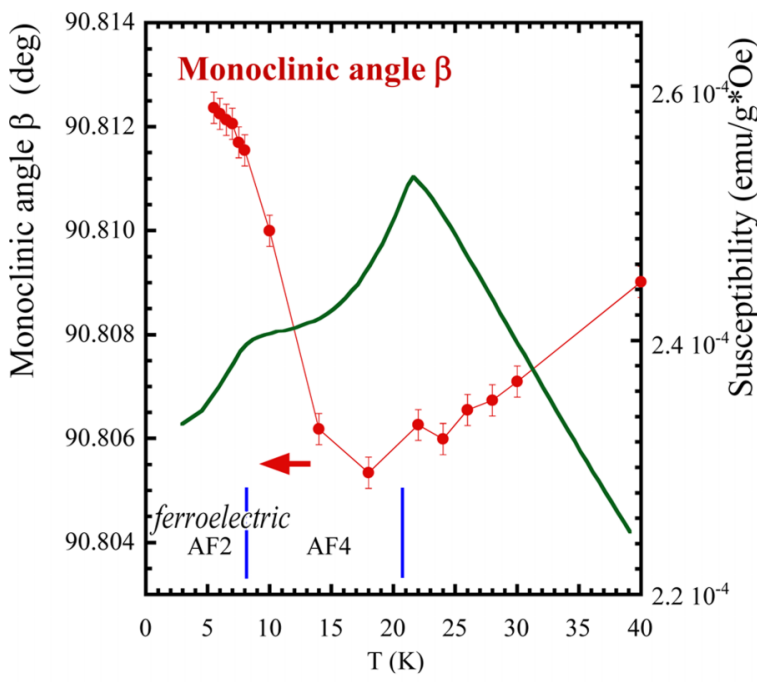

(a)

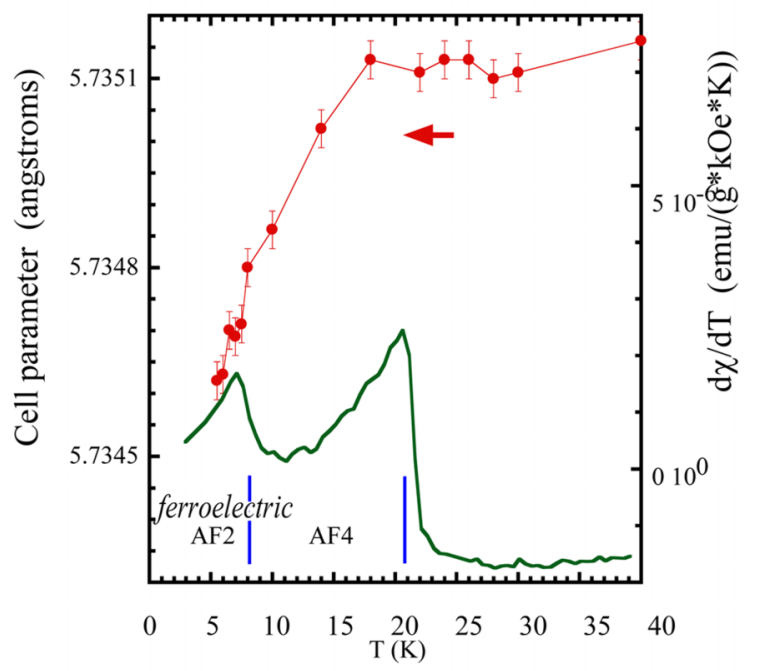

(b)

FIG. 8. Temperature dependence of selected parameters refined from $\mathrm{Mn}_{0.75} \mathrm{Co}_{0.25} \mathrm{WO}_{4}$ synchrotron powder diffraction data. Top panel (a): the monoclinic angle on the left axis (red, dots) and magnetic susceptibility on the right axis (green); bottom panel (b): the b-axis on the left axis (red, dots) and magnetic susceptibility temperature derivative curve on the right axis (green).

highlight the onset of magnetic transitions at $7 \mathrm{~K}$ and $21 \mathrm{~K}$. As expected, structural parameter anomalies were found to match the magnetic transition temperatures, evidencing hence the reliability in collecting data down to a temperature of $5 \mathrm{~K}$.

The final example shows preliminary Extended X-ray Absorption Fine Structure (EXAFS) results obtained at CLÆSS-BL22 on $\mathrm{Ta}_{2} \mathrm{Ni}_{1-\mathrm{x}} \mathrm{Co}_{\mathrm{x}} \mathrm{Se}_{5}$ systems, a complex layered excitonic insulator, in which $\mathrm{Ni}$ single chains and Ta double chains are running along the $a$-axis of the orthorhombic cell, and where tetrahedra and octahedra of Se surround the Ni and Ta ions respectively, while Van der Waals interactions link the planes which are alternately aligned along the $c$-axis ${ }^{17}$ (inset in Fig. 9(c)). The electronic structure of $\mathrm{Ta}_{2} \mathrm{NiSe}_{5}$ is governed by the excitonic coupling between the $\mathrm{Ni} 3 \mathrm{~d}-\mathrm{Se} 4 \mathrm{p}$ hole and the Ta $5 \mathrm{~d}$ electrons at the $\Gamma$ point. ${ }^{18-20}$ Cobalt ions, substituting for the Ni atoms, are expected to strongly modify the physical
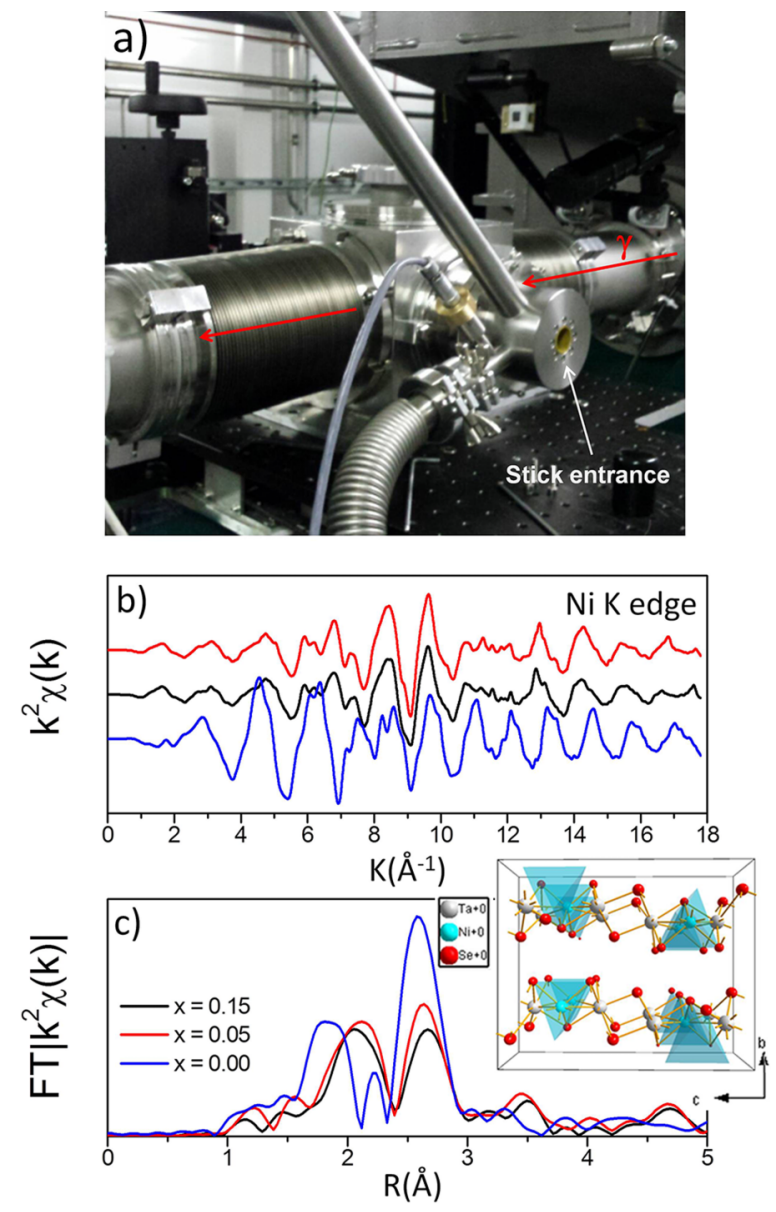

FIG. 9. (a) DynaFlow cryostat installed in the CLÆSS experimental hutch. (b) EXAFS spectra of $\mathrm{Ta}_{2} \mathrm{Ni}_{1-\mathrm{x}} \mathrm{Co}_{\mathrm{x}} \mathrm{Se}_{5}$ (with $\mathrm{x}=0.00,0.05,0.15$ ). (c) Fourier transform of the EXAFS oscillations collected at $27 \mathrm{~K}$. The inset shows the sample crystal structure.

properties of the material. To further understand the nature of this system, a temperature dependent Ni K edge EXAFS study was performed using the DynaFlow cryostat.

Representative low temperature (27 K) EXAFS spectra and their corresponding Fourier Transform (FT) are reported in Figs. 9(b) and 9(c). The complex structure is reflected by the several frequencies composing the EXAFS oscillation and the relative many shell contributions to the FT. The low temperature improvement of the resolution power becomes precious as it opens up the possibility to extend the analyzable K-scale of the EXAFS spectrum, therefore highlighting the contributions from different scatterers more efficiently.

On the basis of $\mathrm{x}$-ray diffraction data, ${ }^{17}$ the first FT peak at low $\mathrm{R}(\mathrm{R} \sim 2-2.5 \AA)$ is given by the 4 chalcogen $(\mathrm{Se})$ atoms which are the first neighbors of the photo-absorbing Ni atom. The second peak at higher $\mathrm{R}(\mathrm{R} \sim 2.5-3 \AA$ ) is given by the $4 \mathrm{Ta}$ atoms lying in the chains. The substitution of Co leads to larger $\mathrm{Ni}-\mathrm{Se}, \mathrm{Ni}-\mathrm{Ta}$ bound distances and results in a general increase of the local disorder (as evident from the damping of the oscillations). A more detailed analysis of the obtained results is beyond the aim of the present paper and it will be the subject of a dedicated publication. A limiting factor at CLÆSS comes from the total absorption at low energy of the Torlon IVC. Additionally, for absorption spectroscopy even 


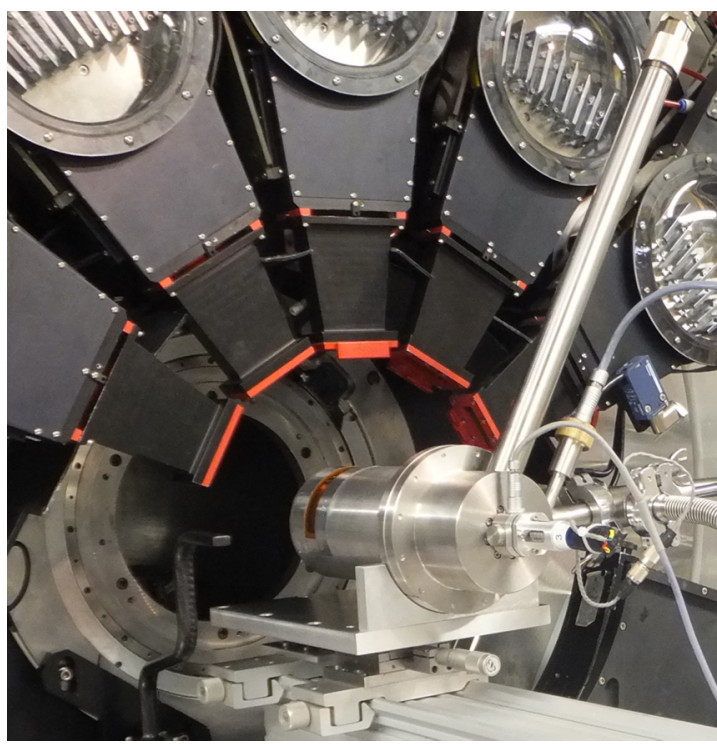

FIG. 10. The DynaFlow cryostat installed in situ on I11.

trace impurities can affect the measurements in fluorescence mode excluding a series of experiments. The demonstration of the impurities in the Torlon dome by XRD renders it unsuitable for CLÆSS. In order to fully take advantage of the available energy range of CLÆSS, a new dome which will host Kapton or glassy carbon windows is currently under investigation.

\section{Use of the DynaFlow cryostat at Diamond Light Source}

The DynaFlow cryostat is currently under commission on the High Resolution Powder Diffraction Beamline I11, where the energy of operation is routinely set at $15 \mathrm{keV} .{ }^{21}$ Previous low temperature capabilities on I11 included the PheniX and the $4 \mathrm{~K}$ cryostat ${ }^{22}$ but both devices offered only a limited rocking angle of the sample, leading to insufficient powder averaging. This is particularly problematic for the parallel beam from the undulator. The new DynaFlow cryostat resolves the particle averaging issue by spinning the capillary at $1 \mathrm{~Hz}$ during data collection, enabling better quality data collection and better resolution of subtle crystallographic changes.

High quality silicon powder is one of the standards used for wavelength calibrations at I11, and the thermal behaviour at low temperatures is well characterised. From previous studies, the general trend is that it has a negative and then positive thermal expansion between 6-120 K and 120-300 K, respectively. ${ }^{23-26}$ Synchrotron powder diffraction measurements on Silicon NIST standard (SRM640d) using the cryostat were performed at wavelength $\lambda=0.825862(9) \AA$ with the sample loaded in a $0.5 \mathrm{~mm}$ diameter borosilicate glass capillary. The crystallographic data are provided by the NIST Certificate. ${ }^{27}$ The capillary was mounted using superglue, centred and attached in the aluminium holder, enabling spinning of the sample up to $1 \mathrm{~Hz}$ whilst collecting data and therefore improving powder averaging. The overall setup, mounting of the cryostat on the diffractometer and sample holder, is displayed in Fig. 10. Using the MAC detectors, 1 h long scans were collected at 4.5, 100, and $295 \mathrm{~K}$.

The data collected from the sample at $\mathrm{T}=4.5 \mathrm{~K}$ were analysed using the TOPAS refinement software ${ }^{28}$ with the input of the published structure of $\mathrm{Si}^{29-31}$ space group $F d \overline{3} m$, lattice parameter, $a=5.43123(8) \AA$ at RT as the starting value. ${ }^{26}$ The data fit was very good as shown in Fig. 11 and the final refined lattice parameter was $a=5.429$ 754(1) $\AA$ for Si. The analysis was repeated for the powder patterns collected at $100 \mathrm{~K}$ and $295 \mathrm{~K}$, and values of $a$ at these temperatures were also obtained (see table in Fig. 11). Data quality is excellent and suitable for Rietveld refinement. The different values of $a$ are consistent with the unusual thermal behaviour of Si. Our preliminary measurements presented here clearly demonstrate the viability of the cryogenic device for high angular resolution diffraction data collected at low cryogenic temperatures. The DynaFlow system will be important for materials research, in particular to study phase transitions caused by thermal or magnetic effects. Future work will also include investigating a new X-ray window to complement the capabilities for time-resolved data collections using the fast position-sensitive detector. ${ }^{32}$ This would enable tracking of fast phase changes (of the order of seconds) in materials which will be important for dynamic studies.

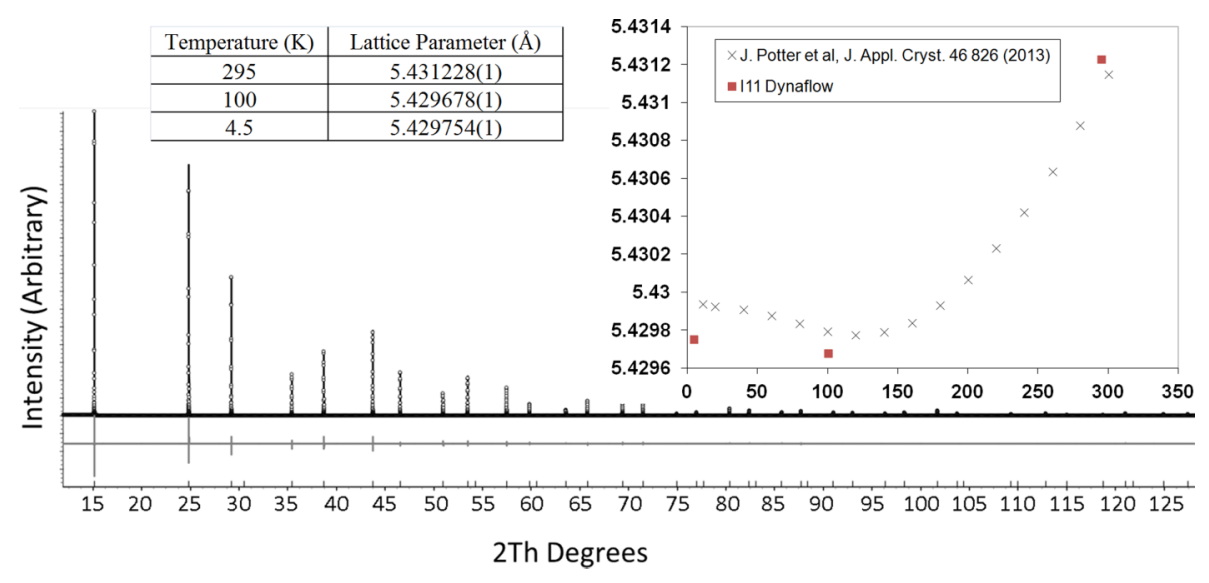

FIG. 11. Refined diffraction pattern at $4.5 \mathrm{~K}$ with the observed data (black line), calculated data (circles), and the difference (grey line underneath the pattern). Table shows the refined lattice parameters for silicon at $295 \mathrm{~K}, 100 \mathrm{~K}$, and $4.5 \mathrm{~K}$ using the DynaFlow cryostat. The inset shows a comparison with previously measured data. ${ }^{22}$ 


\section{CONCLUSIONS}

The dynamic flow cryostat is in routine operation at three different synchrotron research centres. It has proven to be a reliable instrument which is easily adapted for different tender to hard X-ray experimental configurations. Its compact nature, the different accessible working geometries, the efficient and reliable sample cooling and fast sample changing are at the origin of its competitiveness with respect to existing cryostats in the market. When used for temperatures down to $10 \mathrm{~K}$ it has autonomy of more than a week on one standard 1001 Helium Dewar making it particularly well suited for work on synchrotron beamlines. Sample cooling for powder diffraction experiments is efficient at least down to $5 \mathrm{~K}$. Torlon $4203 \mathrm{~L}$ polyamide-imide was used as window material which turned out not to be the best choice for some applications; due to the thickness the transmission was relatively low and impurities present in the material gave a rather large background signal. Further development is required to optimise the windows trading off background signal and impurities versus transmission, especially at low X-ray energies, and life time under radiation.

\section{ACKNOWLEDGMENTS}

PvdL likes to thank C. Malosse for the drafting work and her ideas in improving the mechanics and Ed Mitchell for his help. M.M. would like to thank Ali Al Zein for help on the beamline and Y. Hirata for making the crystals available. F.F. thanks Dr. C. Ritter from Institut Laue Langevin, Grenoble, for supplying the $\mathrm{Tb}_{3} \mathrm{CoNi}$ sample and suggesting the scientific case. Dr. J.-L. Garcia-Munoz is acknowledged for sharing the $\mathrm{Mn}_{0.75} \mathrm{Co}_{0.25} \mathrm{WO}_{4}$ results. J. Prieto, J. Prat, and A. Rizzo from ALBA are acknowledged for the help in the design and the making of the sample spinners. L.S. would like to thank Dr. Wojciech Olszewski for the help in the commissioning and Professor Naurang Saini for the scientific collaboration that made available the samples investigated. C.A.M. and J.P. would like to thank C. Tang for his help.

The cryostats used at ALBA and Diamond Light Source were commercially acquired from the ESRF.

\footnotetext{
${ }^{1}$ See http://www.nist.gov/mml/acmd/structural_materials/cryogenicmatpro p.cfm for NIST cryogenic material properties database.

${ }^{2}$ Selected Cryogenic Data Notebook, compiled and edited by J. E. Jensen, W. A. Tuttle, R. B. Stewart, H. Brechna, and A. G. Prodell, Digitized and put on web from the original Report BNL-10200-R, revised August 1980, https://www.bnl.gov/magnets/staff/gupta/cryogenic-data-handbook/.
}

${ }^{3}$ Torlon PAI design guide, Solvay Advanced Polymers L.L.C. Document EN227547, 2015.

${ }^{4}$ M. Barucci, E. Olivieri, E. Pasca, L. Risegari, and G. Ventura, Cryogenics 45, 295 (2005).

${ }^{5}$ G. Ventura, G. Bianchini, E. Gottardi, I. Peroni, and A. Peruzzi, Cryogenics 39, 481 (1999).

${ }^{6}$ G. P. Robertson, M. D. Guiver, M. Yoshikawa, and S. Brownstein, Polymer 45, 1111 (2004).

${ }^{7}$ B. C. Meyer, Proc. SPIE 8141, 814114 (2011).

${ }^{8}$ M. Moretti Sala, C. Henriquet, L. Simonelli, R. Verbeni, and G. Monaco, J. Electron Spectrosc. Relat. Phenom. 188, 150 (2013).

${ }^{9}$ M. Moretti Sala, K. Ohgushi, A. Al-Zein, Y. Hirata, G. Monaco, and M. Krisch, Phys. Rev. Lett. 112, 176402 (2014).

${ }^{10}$ F. Fauth, I. Peral, C. Popescu, and M. Knapp, Powder Diffr. 28, S360 (2013).

${ }^{11}$ F. Fauth, R. Boer, F. Gil-Ortiz, C. Popescu, O. Vallcorba, I. Peral, D. Fulla, J. Benach, and J. Juanhuix, Eur. Phys. J. Plus 130, 160 (2015).

${ }^{12}$ T. B. Massalski, Binary Alloy Phase Diagrams, 2nd ed. (ASM International, Materials Park, Ohio, 1990), ISBN: 978-0-87170-403-0.

${ }^{13}$ P. Villars and K. Cenzual, Pearson's Crystal Data-Crystal Structure Database for Inorganic Compounds, Release 2010/11, ASM International, Materials Park, Ohio, 2010/11.

${ }^{14} \mathrm{C}$. Ritter (unpublished).

${ }^{15}$ I. Urcelay-Olabarria, J. L. Garcia-Munoz, E. Ressouche, V. Skumryev, V. Yu. Ivanov, A. A. Mukhin, and A. M. Balbashov, Phys. Rev. B 86, 184412 (2012).

${ }^{16}$ J. Rodriguez-Carvajal, Physica B 192, 55 (1993).

${ }^{17}$ S. A. Sunshine and J. A. Ibers, Inorg. Chem. 24, 3611 (1985).

${ }^{18}$ Y. Wakisaka, T. Sudayama, K. Takubo, T. Mizokawa, M. Arita, H. Namatame, M. Taniguchi, N. Katayama, M. Nohara, and H. Takagi, Phys. Rev. Lett. 103, 026402 (2009).

${ }^{19}$ T. Kaneko, T. Toriyama, T. Konishi, and Y. Ohta, J. Phys.: Conf. Ser. 400, 032035 (2012).

${ }^{20}$ K. Seki, Y. Wakisaka, T. Kaneko, T. Toriyama, T. Konishi, T. Sudayama, N. L. Saini, M. Arita, H. Namatame, M. Taniguchi, N. Katayama, M. Nohara, H. Takagi, T. Mizokawa, and Y. Ohta, Phys. Rev. B 90, 155116 (2014).

${ }^{21}$ S. P. Thompson, J. E. Parker, J. Potter, T. P. Hill, A. Birt, T. M. Cobb, F. Yuan, and C. C. Tang, Rev. Sci. Instrum. 80, 075107 (2009).

${ }^{22}$ J. Potter, J. E. Parker, A. R. Lennie, S. P. Thompson, and C. C. Tang, J. Appl. Crystallogr. 46, 826 (2013).

${ }^{23}$ D. N. Batchelder and R. O. Simmons, J. Chem. Phys. 41, 2324 (1964).

${ }^{24}$ J. S. Shah and M. Straumanis, Solid State Commun. 10, 159 (1972).

${ }^{25}$ R. R. Reeber and K. Wang, Mater. Chem. Phys. 46, 259 (1996).

${ }^{26}$ K. G. Lyon, G. L. Salinger, C. A. Swenson, and G. K. White, J. Appl. Phys. 48, 865 (1977).

${ }^{27}$ National Institute of Standards and Technology Certificate Standard Reference Material $®$ 640d Silicon Powder Line Position and Line Shape Standard for Powder Diffraction, 26th May 2010.

${ }^{28}$ Bruker AXS Diffrac.suite TOPAS 4.2, Bruker AXS GmbH, Karlruhe, Germany. Technical reference: DOC-M88-EXX066 V4.2. Updated Jan 15, 2009.

${ }^{29}$ A. Bergamin, G. Cavagnero, G. Mana, and G. Zosi, J. Appl. Phys. 82, 5396 (1997).

${ }^{30}$ H. Kuestner and H. Remy, Phys. Z. 24, 25 (1923).

${ }^{31}$ P. Debye and P. Scherrer, Phys. Z. 17, 277 (1916).

${ }^{32}$ S. P. Thompson, J. E. Parker, J. Marchal, J. Potter, A. Birt, F. Yuan, R. D. Fearn, A. R. Lennie, S. R. Street, and C. C. Tang, J. Synchrotron Radiat. 18, 637 (2011). 